\title{
LERAY RESIDUE FOR SINGULAR VARIETIES
}

\author{
ANDRZEJ WEBER \\ Institute of Mathematics, University of Warsaw \\ ul. Banacha 2, 02-097 Warszawa, Poland \\ E-mail: aweber@mimuw.edu.pl
}

1. Residue form. We begin with recalling the construction of the Leray residue form [Le]. Let $K$ be a smooth hypersurface of a complex manifold $M$. Suppose that $K$ is locally given by an equation $f=0$ where $f$ is holomorphic and $d f$ does not vanish along $K$. Let $\omega \in \Omega^{k+1}(M \backslash K)$ be a complex valued $C^{\infty}$-form with the first order pole on $K$, i.e. $f \omega$ extends to a smooth form on $M$. If $\omega$ is closed then it can be written (locally) in the form

$$
\omega=\frac{d f}{f} \wedge r+\theta,
$$

where $r$ and $\theta$ are $C^{\infty}$-forms on $M$. A tool to obtain this decomposition is the Division Property:

Definition. Suppose $d f \neq 0$ at $x \in M$. The differential $d f$ is said to have a Division Property if for every smooth form $\alpha$ satisfying $d f \wedge \alpha=0$, there exists a smooth form $\beta$ defined on a neighbourhood of $x$ such that $\alpha=d f \wedge \beta$.

The Leray residue form of $\omega$ is defined by

$$
\operatorname{Res} \omega=r_{\mid K} .
$$

The form $r_{\mid K}$ does not depend on $f$ nor on the decomposition of $\omega$, thus $\operatorname{Res} \omega \in \Omega^{k}(K)$ is defined globally. The form Res $\omega$ is closed. Its class in $H^{k}(K)$ depends only on the class of $\omega$ in $H^{k+1}(M \backslash K)$.

Consider now a singular hypersurface $K=\{f=0\}$. To construct the residue form we have to use the Division Property of a singular differential $d f$. Suppose $\omega$ is a meromorphic $(k+1)$-form with the first order pole on $K$. To decompose $\omega$ and obtain a residue form

Partially supported by KBN grant 2 P03A 01113.

1991 Mathematics Subject Classification: Primary 32S20, 55N33; Secondary 14C30, 32S40.

Received by the editors: November 27, 1997; in the revised form: January 12, 1998.

The paper is in final form and no version of it will be published elsewhere. 
(as in the smooth case) we have to use the Division Property twice: for holomorphic $(k+2)$ - and $(k+1)$-forms. The differential $d f$ has the Division Property for holomorphic $l$-forms $\alpha$, provided that $l$ is not greater than the codimension of singularities $\Sigma_{K}=\{f=0, d f=0\}$ in $K$ as proved in [Sa] (see also [dR] for isolated singularities). For example, if $K$ has isolated singularities then the division is possible for $l$-forms with $l \leq n$ where $n=\operatorname{dim}_{\mathbb{C}} K$. Thus one can construct the residue form for $\omega$ of degree smaller than $n$. The residue forms are well defined on the nonsingular part of $K$. In general they have poles at singular points $\Sigma_{K}$. Consider an example:

ExAmPle 1.1. Let $M=\mathbb{C}^{3}, f(x, y, z)=x^{3}+y^{3}+z^{3}, \omega=\frac{1}{f} d x \wedge d y \wedge d z$, then

$$
\operatorname{Res} \omega= \begin{cases}\frac{1}{3 x^{2}} d y \wedge d z & \text { for } x \neq 0 \\ -\frac{1}{3 y^{2}} d x \wedge d z & \text { for } y \neq 0 \\ \frac{1}{3 z^{2}} d x \wedge d y & \text { for } z \neq 0 .\end{cases}
$$

A possibility to find a residue class in $H^{*}(K)$ from the integration point of view means to give a meaning to the symbol

$$
\int_{\xi} \operatorname{Res} \omega
$$

for a cycle $\xi$ which is allowed to intersect singularities. A question how to give a meaning to this symbol was asked by Prof. Bogdan Ziemian. It arises in a natural way from his calculation $[\mathrm{Zi}]$.

We are mainly interested in the residues of holomorphic $(n+1)$-forms. The purpose of this paper is to show a sequence of local conditions for the singularities of $K$ which allow to lift the homological residue to cohomology or to intersection homology. We will proceed as follows. After discussion on the topological point of view in Section 2 we investigate $K$ with isolated singularities. We consider a sequence of singularity types. Each one is worse than the preceding one. We begin with rational homology manifolds in Section 3, next we describe a condition on the oscillation index in Section 4 and in Section 5 we give a condition for the spectrum of a singular point. Finally we find in Section 6 the obstruction to lift, called the second residue. Later we discuss nonisolated singularities: line singularities in Section 7 and the case when $K$ is a sum of smooth components with normal crossings in Section 8. We finish with a remark for the general case in Section 9.

This paper was stimulated by discussions with Prof. Bogdan Ziemian, who investigated residues by means of hyperfunctions. Prof. Bogdan Ziemian died tragically in the spring of 1997. First of all I would like to thank him for setting the problem and for the help with finding the solution. There are other people who influenced my work. They are Professors G. Barthel, J-P. Brasselet, B. Jakubczyk, P. Jaworski and H. Żołądek. The detailed discussion and complete proofs of the presented theorems will appear in [We2].

2. Topological point of view. Suppose $M^{n+1}$ is a complex manifold and $K^{n}$ is a hypersurface. Let $c \in H^{k+1}(M \backslash K)$ be a cohomology class. Suppose for a moment that $K$ is nonsingular. Consider a diagram of cohomology and homology groups with coefficients 
in $\mathbb{C}$ :

$$
\begin{aligned}
& H^{k+1}(M \backslash K) \stackrel{\delta}{\longrightarrow} H^{k+2}(M, M \backslash K) \quad=\quad H^{k+2}(\operatorname{Tub} K, \operatorname{Tub} K \backslash K) \\
& \text { Poincaré duality } \downarrow \cap[M] \quad \tau \uparrow \text { Thom isomorphism } \\
& H_{2 n-k}^{B M}(K) \quad \stackrel{\cap[K]}{\text { Poincaré duality }} \quad H^{k}(K)
\end{aligned}
$$

In the diagram $H_{*}^{B M}$ denotes Borel-Moore homology, i.e. homology with closed supports. For simplicity we will assume that $K$ is compact, then $H_{*}^{B M}$ is ordinary homology. Using Thom isomorphism we can construct the residue of the class $c$ :

$$
\text { res } c=2 \pi i \tau^{-1} \delta c=2 \pi i(\cap[K])^{-1}(\delta c \cap[M]) \in H^{k}(K) .
$$

If $c$ is represented by $\omega \in \Omega^{k+1}(M \backslash K)$ with the first order pole on $K$ then

$$
[\operatorname{Res} \omega]=\operatorname{res} c
$$

by [Le]. If $K$ is singular then the Poincaré duality map

$$
P D: H^{k}(K) \stackrel{\cap[K]}{\longrightarrow} H_{2 n-k}(K)
$$

does not have to be an isomorphism. We can still define a residue class of $c$, but this time in homology

$$
\operatorname{res} c:=2 \pi i \delta c \cap[M] \in H_{2 n-k}(K) .
$$

We call it the homological residue. If $K$ is singular then not every class res $c$ lies in the image of $P D$.

For $K$ with isolated singularities (for $\operatorname{dim}_{\mathbb{C}} K=n>1$ ) the image of the Poincaré duality map is the intersection homology group (with middle perversity) $I H_{n}(K)$. In general this is not true, the definition of this group is more sophisticated (see [GM]). In any case the map $P D$ factors through intersection homology:

$$
\begin{array}{ccc}
\alpha \nearrow & I H_{2 n-k}(K) & \searrow^{\beta} \\
H^{k}(K) & P D & H_{2 n-k}(K) .
\end{array}
$$

We will ask about the existence of a lift $\beta^{-1}(\operatorname{res} c)$ of the homological residue to intersection homology. This question seems to be more natural and a positive answer is more often possible.

Let $K^{\circ}$ be a manifold with boundary obtained from $K$ by removing small balls centred at singular points. The boundary $\partial K^{\circ}$ is the sum of links of the singular points. Suppose $n>1$. In the middle dimension we have

$$
H^{n}(K) \simeq H^{n}\left(K^{\circ}, \partial K^{\circ}\right)
$$

and by Poincaré duality for $K^{\circ}$

$$
H_{n}(K) \simeq H_{n}\left(K^{\circ}, \partial K^{\circ}\right) \simeq H^{n}\left(K^{\circ}\right) .
$$

The Poincaré duality map $P D$ coincides with the natural map of cohomology induced by the inclusion $\left(K^{\circ}, \emptyset\right) \subset\left(K^{\circ}, \partial K^{\circ}\right)$. Consider the cohomology long exact sequence of the second pair. We have

$$
\operatorname{im}(P D)=\operatorname{im}\left(H^{n}\left(K^{\circ}, \partial K^{\circ}\right) \rightarrow H^{n}\left(K^{\circ}\right)\right)=\operatorname{ker}\left(H^{n}\left(K^{\circ}\right) \rightarrow H^{n}\left(\partial K^{\circ}\right)\right),
$$


where the maps are the natural ones induced by inclusions. To show that the homological residue

$$
\operatorname{res}[\omega]=[\operatorname{Res} \omega] \in H^{n}\left(K^{\circ}\right) \simeq H_{n}(K)
$$

lies in the image of $P D$ it is necessary and sufficient to show that

$$
\left[\operatorname{Res} \omega_{\mid L_{x}}\right]=0
$$

for the link $L_{x}$ of any singular point $x \in K$. By the de Rham theorem we obtain a criterion:

Proposition 2.1. If $\int_{\xi} \operatorname{Res} \omega=0$ for any cycle $\xi$ contained in the link $L_{x}$, then $[\operatorname{Res} \omega]$ belongs to the image of $P D$.

Note that the integral is well defined since $\xi$ does not intersect singularities.

3. Rational homology manifolds. We remind that a rational homology manifold is a space such that the link of any point is a rational homology sphere. From the homological point of view such spaces possess properties of the ordinary manifolds.

We want to invert the Poincaré duality map

$$
P D: H^{k}(K) \stackrel{\cap[K]}{\longrightarrow} H_{2 n-k}(K)
$$

or at least to show that an element res $c \in H_{2 n-k}(K)$ lies in the image. Obviously if $K$ is a rational homology manifold then the map $P D$ is an isomorphism.

Suppose $K$ has isolated singularities, then there is a criterion examining if $K$ is a rational manifold. Consider the Milnor fibration of each singular point and the monodromy acting on the vanishing cycles (i.e. on the homology of the Milnor fibre).

Theorem 3.1 [Mi]. A variety $K$ is a rational homology manifold if and only if 1 is not an eigenvalue of the monodromy of any singular point.

Among $K$ with simple singularities the rational homology manifolds are those with the following singularities: $A_{2 k}, A_{2 k+1}\left(n\right.$ odd), $D_{k}$ ( $n$ even), $E_{6}, E_{7}\left(n\right.$ even), $E_{8}$.

4. Positive oscillation index. Let $K$ be a hypersurface with isolated singularities. For simplicity we assume that $\operatorname{dim}_{\mathbb{C}} K=n>1$. In Sections $4-7$ we consider only the holomorphic forms of the type $(n+1,0)$ with a first order pole on $K$. Then the homological residue belongs to the middle dimension group $H_{n}(K)$.

Suppose that $K$ is described by a function $f$. Fix $\delta \ll \epsilon \ll 1$ such that $f$ restricted to $B_{\epsilon} \cap f^{-1}\left(\dot{D}_{\delta}\right)$ is a fibration over $\dot{D}_{\delta}$ (here $B_{\epsilon}$ is the ball centred in a singular point $x$ and $\dot{D}_{\delta}$ is the punctured disk around 0). The intersection $S_{\epsilon} \cap f^{-1}(0)=\partial B_{\epsilon} \cap f^{-1}(0)$ is the link $L_{x}$ of the singular point.

We will recall a construction of oscillating integrals. Let $\eta$ be a holomorphic $(n+1)$ form. For any (possibly multivalued) continuous family of cycles $\zeta_{t} \subset B_{\epsilon} \cap f^{-1}(t), t \in \dot{D}_{\delta}$ consider the integral

$$
I(t)=\int_{\zeta_{t}} \eta / d f .
$$


The function $I(t)$ is (multivalued) holomorphic and expands as ([AGV, §11])

$$
I(t)=\sum_{\alpha \in \mathbb{Q}, k \in \mathbb{N}} a_{\alpha, k} t^{\alpha}(\ln t)^{k}
$$

Consider the set of exponents in the expansions of $I(t)$ for any $\eta$ and $\zeta_{t}$. Some of the exponents that are not greater than 0 coincide with the spectral numbers of the singularity. References for the spectrum are $[\mathrm{AGV}, \S 13.3]$ and [St]. The numbers $\exp (2 \pi i \alpha)$, where $\alpha$ is in the spectrum, are the eigenvalues of the monodromy. Note that if $K$ is a rational homology manifold then 1 is not an eigenvalue and thus no integer is a spectral number. The spectral numbers which are not greater than 0 can be read from the Newton diagram for nondegenerated function $f([\mathrm{AGV}, \mathrm{p} .273])$.

Let $\alpha_{\min }$ be the smallest number occurring among all exponents of the expansions of $I(t)$ for families $\zeta_{t}$ and forms $\eta$. It is a rational number greater than -1 , called the oscillation index of the critical point.

Now we come back to our problem. Let $\xi \subset L_{x}$ be a cycle. Note that the function $f$ restricted to $S_{\epsilon} \cap f^{-1}\left(D_{\delta}\right)$ is a trivial fibration over the whole disk. Thus the cycle $\xi$ can be contained in a continuous family of cycles $\xi_{t} \subset S_{\epsilon} \cap f^{-1}(t), t \in D_{\delta}$ with $\xi_{0}=\xi$. The form $\omega$ can be written as $\omega=\frac{\eta}{f}$ with $\eta$ holomorphic. (This exactly means that the order of pole of $\omega$ is not greater than 1.) Then

$$
\operatorname{Res} \omega=(\eta / d f)_{\mid\{f=0\}}
$$

and the form $\eta / d f$ is well defined after restriction to each level set. We will examine when $\int_{\xi} \operatorname{Res} \omega=0$ according to the Proposition 2.1. Let us move the cycle $\xi$ slightly away from $K$ and see what is the asymptotic of the integral

$$
I(t)=\int_{\xi_{t}} \eta / d f
$$

as the cycle $\xi_{t}$ approaches $\xi$. This integral converges to $\int_{\xi} \operatorname{Res} \omega$. The family $\xi_{t}$ is single valued and extends over the whole disk, thus the function $I(t)$ is holomorphic. If all the exponents are positive in the expansion of $I(t)$ then $I(0)=0$. We obtain:

Proposition 4.1. Suppose $\alpha_{\min }>0$, then for any cycle $\xi \subset L_{x}$ we have

$$
\int_{\xi} \operatorname{Res} \omega=\int_{\xi_{0}} \eta / d f=I(0)=0 .
$$

COROLlary 4.2. Suppose the oscillation indices of each singular point of $K$ are positive, then the homological residue of any form lifts to cohomology.

Let us consider a special type of singularities, namely quasihomogeneous singularities. We assume that in a neighbourhood of each singular point of $K$ there exists a coordinate system such that $K$ is defined by a function $f_{x}$ which is homogeneous with respect to the weights $a_{0}, a_{1}, \ldots a_{n}$. We may assume that $\operatorname{deg} f_{x}=1$. Then the oscillation index is

$$
\alpha_{\min }=a_{0}+a_{1}+\ldots+a_{n}-1 .
$$

Let us introduce the canonical metric on the cone over a manifold $L_{x}$. It is defined on the nonsingular part $c L_{x} \backslash\{$ vertex $\}=L_{x} \times(0,1)$ by the formula

$$
t^{2} d x \otimes d x+d t \otimes d t,
$$


where $x$ is the coordinate in the base and $t$ is the parameter of $(0,1)$. We say that a Riemannian metric on $K \backslash \Sigma_{K}$ is conelike if $K$ is locally isometric to the metric cone over the link. This means that we assume that there are homeomorphism of neighbourhoods of singular points with the cones over links: $U_{x} \simeq c L_{x}$ which are isometries on the nonsingular parts. Under assumption that $K$ has quasihomogeneous singularities with positive oscillation indices we can prove

TheOREM 4.3 [We2]. Suppose that $K$ is described in the neighbourhoods of the singular points by quasihomogeneous functions with $\alpha_{\min }>0$. Let $p \geq 2$ be a real number. Then there exists a conelike metric on $K$ such that the norm of the residue form is a function integrable in the p-th power, i.e.

$$
|\operatorname{Res} \omega| \in L^{p}\left(K \backslash \Sigma_{K}\right)
$$

This way we see that $[\operatorname{Res} \omega]$ defines an element in $L_{p}$-cohomology of $K \backslash \Sigma_{K}$. This group is isomorphic to intersection homology for $p \in\left[2,2+\frac{2}{n-1}\right)$ and to cohomology for $p \geq 2+\frac{2}{n-1}([\mathrm{We} 1])$.

Practically this means that the residue form is integrable on the cycles which enter singularities in a regular way, i.e. along cone lines.

5. Negative oscillation index. Suppose now that the condition $\alpha_{\min }>0$ is not fulfilled. We can still formulate a condition allowing to lift the homological residue to cohomology.

THEOREM 5.1. The condition " 0 is not a spectral number of any singular point of $K$ " is a necessary condition for the existence of lifting of homological residue to cohomology. It is also sufficient for $K$ with quasihomogeneous singularities.

Theorem 5.1 follows from the observation that

$$
\int_{\xi} \operatorname{Res} \omega=\int_{\xi_{0}} \eta / d f=I(0)=a_{0,0} .
$$

For quasihomogeneous singularities the considered spectral numbers are of the form

$$
\sum_{i=0}^{n} k_{i} a_{i}-1, \quad \text { where each } k_{i}>0 \text { is a natural number. }
$$

The example of a singularity with 0 in the spectrum is any homogeneous singularity with $\operatorname{deg} f \geq n+1$. If $\operatorname{deg} f=n+1$ then $\alpha_{\min }=0$. On the other hand we have numerous examples of polynomials with $\alpha_{\min }<0$ for which 0 is not in the spectrum, e.g.

$$
f=z_{0}^{2}+z_{1}^{4}+z_{2}^{5}
$$

with the set of exponents $-\frac{1}{20}=\alpha_{\min }, \frac{3}{20}, \frac{4}{20}, \ldots$.

For quasihomogeneous singularities there is an elementary proof of the Theorem 5.1 which does not involve the theory of oscillating integrals. It can be found in [We2].

6. The second residue. Suppose that 0 is a spectral number. Then a local obstruction to lift occurs. This is the element $\left[\operatorname{Res} \omega_{\mid L_{x}}\right] \in H^{n}\left(L_{x}\right)$. If the singularity is quasihomogeneous then we have an action of $S^{1}$ on the link. The quotient $L_{x} / S^{1}$ is a 
subset of the weighted projective space. It is a $V$-manifold ([St]), it behaves like a smooth projective variety from the point of view of Hodge theory. Integrating the residue form over the orbits of the $S^{1}$ action we obtain a holomorphic $(n-1)$-form on $L_{x} / S^{1}$. We call it the second residue.

Theorem 6.1 [We2]. Suppose $\omega=\frac{g}{f} d z_{0} \wedge \cdots \wedge d z_{n}$. Let $\operatorname{res}_{2} \omega \in H^{n-1}\left(L_{x} / S^{1}\right)$ be the second residue of $\omega$. Then the following conditions are equivalent:

1) $\operatorname{res}_{2} \omega=0 \in H^{n-1}\left(L_{x} / S^{1}\right)$;

2) $\left[\operatorname{Res} \omega_{\mid L_{x}}\right]=0 \in H^{n}\left(L_{x}\right)$;

3) the quasihomogeneous component of $g$ with weight $-\alpha_{\min }$ vanishes.

Of course if all local obstructions vanish then the homological residue lifts to intersection homology.

Consider the $P_{8}$ singularity (Example 1.1): $f=z_{0}^{3}+z_{1}^{3}+z_{2}^{3}$. The affine part of $L_{0} / S^{1}$ is

$$
\left\{1+u_{1}^{3}+u_{2}^{3}=0\right\} \subset \mathbb{C}^{2} \subset \mathbb{P}^{2} .
$$

We have

$$
\operatorname{res}_{2}\left(\frac{1}{f} d z_{0} \wedge d z_{1} \wedge d z_{2}\right)=\left[u_{1} d u_{2}-u_{2} d u_{1}\right]
$$

Its class in $H^{1}\left(L_{0} / S^{1}\right)$ is nontrivial.

7. Line singularities. Suppose now that the singular locus of $K$ is of dimension one. Taking a transversal slice of $\Sigma_{K}$ in each generic point we obtain a family of isolated singularities. The cohomology of the link is a local system over $\Sigma_{K}$ minus a discrete set of points. In each slice we obtain a second residue - the obstruction to lift. Instead of describing the general situation we consider some examples.

EXAMPLE 7.1. The degeneration of self-intersections:

$$
f=x^{2}\left(x+z^{2}\right)-y^{2} .
$$

We treat $z$ as a parameter. The singular locus is $\Sigma_{K}=\{x=y=0\}$. Let

$$
\omega=\frac{1}{f} d x \wedge d y \wedge d z=\frac{d f}{f} \wedge \frac{d x \wedge d z}{2 y} .
$$

We have $y= \pm x \sqrt{x+z^{2}}$ on $K$. Thus

$$
\operatorname{Res} \omega=\frac{d x}{x} \wedge \frac{d z}{ \pm 2 \sqrt{x+z^{2}}} .
$$

The residue form has a pole in the self-intersection point $x=y=0$. The second residue is

$$
\operatorname{Res}_{2} \omega= \pm \frac{d z}{2 \sqrt{x+z^{2}}}= \pm \frac{d z}{2 z} .
$$

This form again has a pole. The resulting residue may be called the third residue. In general we can build the whole sequence of residues supported by the strata of a stratification.

EXAMPLE 7.2. A family of intersecting lines-Whitney umbrella:

$$
f=z x^{2}-y^{2} \text {. }
$$


Again $\Sigma_{K}=\{x=y=0\}, \omega=\frac{1}{f} d x \wedge d y \wedge d z$. Then $y= \pm x \sqrt{z}$ on $K$ and

$$
\operatorname{Res} \omega=\frac{d x}{x} \wedge \frac{d z}{2 y}=\frac{d x}{x} \wedge \frac{d z}{ \pm 2 \sqrt{z}} .
$$

We obtain the second residue $\operatorname{Res}_{2} \omega= \pm \frac{d z}{2 \sqrt{z}}$ which is an essentially multivalued form. Indeed the bundle of the cohomology of the links for $z \neq 0$ is twisted. We take a double covering of $\mathbb{C} \backslash\{0\}$ by substitution $z=\tilde{z}^{2}$. We obtain a nonsingular second residue $d \tilde{z}$.

8. Smooth divisor with normal crossings. Suppose $K$ is locally described by the function

$$
f=z_{0} z_{1} \cdots z_{l} \quad l \leq n .
$$

Then $K$ is a sum of irreducible $K_{i}$ 's. If we assume (as we do) that each $K_{i}$ has no self-intersection then $K_{i}$ is smooth.

One defines the subcomplex of $\Omega^{*}(M \backslash K)$ consisting of the forms having only logarithmic singularities on $K$. It is denoted by $\Omega^{*}(M, \log \langle K\rangle)$. Locally it is spanned by $\frac{d z_{0}}{z_{0}}, \ldots \frac{d z_{l}}{z_{l}}$ over $\Omega^{*}(M)$. It has the same cohomology as $\Omega^{*}(M \backslash K)$. There is an increasing filtration of $\Omega^{*}(M, \log \langle K\rangle)$ introduced by Deligne [De], [GS]:

$$
\mathcal{W}_{i}=\Omega^{*-i}(M) \wedge \Omega^{i}(M, \log \langle K\rangle) .
$$

In fact the Deligne weight filtration $W_{*}$ is slightly translated: $W_{i+k} \Omega^{k}(M, \log \langle K\rangle)=\mathcal{W}_{i}$, but for us the notation $\mathcal{W}_{*}$ (as in [GS]) is more convenient.

Let $K^{[i]}$ be the disjoined union of $i$-fold intersections of $K_{j}$ 's:

$$
K^{[i]}=\bigsqcup_{j_{1}<\ldots<j_{i}} K_{j_{1}} \cap \ldots \cap K_{j_{i}} .
$$

The iterated residue defines a map

$$
\operatorname{Res}_{i}: \mathcal{W}_{i} / \mathcal{W}_{i-1} \rightarrow \Omega^{*-i}\left(K^{[i]}\right)
$$

which passes to an isomorphism of cohomology ([GS])

$$
\operatorname{res}_{i}: H^{*}\left(\mathcal{W}_{i} / \mathcal{W}_{i-1}\right) \stackrel{\simeq}{\rightarrow} H^{*-i}\left(K^{[i]}\right) .
$$

In particular if $\omega \in \mathcal{W}_{0}$ then $\omega$ has no pole along $K$ and $\operatorname{Res} \omega=0$. If $\omega \in \mathcal{W}_{1}$ is a $(k+1)$-form then we obtain the first residue, which is a $k$-form on each component:

$$
\operatorname{res}_{1}[\omega] \in \bigoplus_{j} H^{k}\left(K_{j}\right)=I H_{2 n-k}(K) .
$$

EXAmPLE 8.1. Let $M=\mathbb{C}^{2}, f=x y, \omega=\frac{a x+b y}{x y} d x \wedge d y=d x \wedge a \frac{d y}{y}+b \frac{d x}{x} \wedge d y \in \mathcal{W}_{1}$. Then

$$
\operatorname{Res} \omega= \begin{cases}b d y & \text { for } x=0 \\ -a d x & \text { for } y=0 .\end{cases}
$$

In general if $\omega \in \mathcal{W}_{i} \backslash \mathcal{W}_{i-1}$, then all the residue forms $\operatorname{Res}_{k} \omega$ are singular for $k<i$ and $\operatorname{Res}_{i} \omega$ is nonsingular on $K^{[i]}$.

EXAMPLE 8.2. Let $M=\mathbb{C}^{2}, f(x, y)=x y, \omega=\frac{1}{f} d x \wedge d y \in \mathcal{W}_{2}$, then

$$
\operatorname{Res} \omega= \begin{cases}\frac{d y}{y} & \text { for } x=0, y \neq 0 \\ -\frac{d x}{x} & \text { for } y=0, x \neq 0 .\end{cases}
$$


We obtain the second residue $\operatorname{res}_{2}[\omega]= \pm 1 \in H^{0}(\{0,0\})$.

Suppose $M$ is a projective manifold. Then $H^{*}(M \backslash K)$ is equipped with a mixed Hodge structure. Thus $H^{*}(M \backslash K)$ can be canonically decomposed ([GS, 1.12]):

$$
H^{*}(M \backslash K)=\bigoplus_{j \geq 0} \overline{\mathcal{W}}_{j}
$$

such that $\boldsymbol{W}_{i}=\bigoplus_{i \geq j \geq 0} \overline{\mathcal{W}}_{j}$ is the filtration induced by $\mathcal{W}_{i}$ on $H^{*}(M, \backslash K)$. This way we can associate with a class $c \in H^{*}(M \backslash K)$ a sequence of residues in $\overline{\mathcal{W}}_{j}$. Unfortunately $\overline{\mathcal{W}}_{j}$ is only a quotient of a subspace in $H^{*}\left(K^{[j]}\right)$, so we cannot find a canonical element in $H^{*}\left(K^{[j]}\right)$.

9. Remark for a general case. Suppose $M$ is algebraic. To define weights on $M \backslash K$ we resolve $K$ to obtain $\widetilde{K} \subset \widetilde{M}$, a smooth divisor with normal crossings. If $c \in H^{k}(M \backslash K)=H^{k}(\widetilde{M} \backslash \widetilde{K})$ belongs to $\mathcal{W}_{1}$ then we have $\operatorname{res}_{1} c \in I H_{2 n-k+1}(\widetilde{K})$. Now we can use functoriality of intersection homology ([BBFGK], [We3]). There exists a morphism $\mu$ (in general not unique) completing the diagram

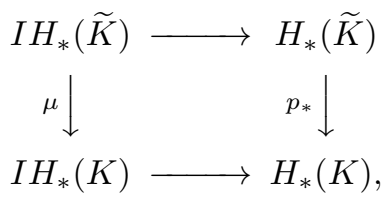

where $p: \widetilde{K} \rightarrow K$ is a desingularization. The element $\mu(\operatorname{res} c) \in I H_{2 n-k+1}(K)$ is the desired lift of homological residue to intersection homology.

\section{References}

[AGV] V. I. Arnol'd, S. M. Guseŭn-Zade, A. N. Varchenko, Singularities of Differentiable Mappings II, Nauka, Moscow, 1984 (Russian).

[BBFGK] G. Barthel, J.-P. Brasselet, K.-H. Fieseler, O. Gabber, L. Kaup, Relèvement de cycles algébriques et homomorphismes associés en homologie d'intersection, Ann. of Math. (2) 141 (1995), 147-179.

[De] P. Deligne, Poids dans la cohomologie des variétés algébriques, in: Proceedings of the International Congress of Mathematicians (Vancouver, 1974), vol. 1, Canad. Math. Congress, Montreal, 1975, 79-85.

[GM] M. Goresky, R. MacPherson, Intersection homology II, Invent. Math. 72 (1983), $77-129$.

[GS] P. Griffiths, W. Schmid, Recent developments in Hodge theory: a discussion of techniques and results, in: Discrete Subgroups of Lie Groups and Applications to Moduli (Internat. Colloq., Bombay, 1973), Oxford Univ. Press, Bombay, 1975, $31-127$.

[Le] J. Leray, Le calcul différentiel et intégral sur une variété analytique complexe (Problème de Cauchy, III), Bull. Soc. Math. France 87 (1959), 81-180.

[Mi] J. Milnor, Singular Points of Complex Hypersurfaces, Princeton Univ. Press, Princeton, 1968. 
[dR] G. de Rham, Sur la division de formes et de courants par une forme linèaire, Comment. Math. Helv. 28 (1954), 346-352.

[Sa] K. Saito, On a generalization of de-Rham lemma, Ann. Inst. Fourier (Grenoble) 26 (1976), 165-170.

[St] J. H. M. Steenbrink, The spectrum of hypersurface singularities, Astérisque 179-180 (1989), 163-184.

[We1] A. Weber, An isomorphism from intersection homology to $L_{p}$-cohomology, Forum Math. 7 (1995), 489-512.

[We2] A. Weber, Leray residue form and intersection homology, preprint.

[We3] A. Weber, A morphism of intersection homology induced by an algebraic map, Proc. Amer. Math. Soc., to appear.

[Zi] B. Ziemian, Leray residue formula and asymptotics of solutions to constant coefficients PDEs, Topol. Methods Nonlinear Anal. 3 (1994), 257-293. 